\title{
Environment Estimation in Teleoperation Systems
}

\author{
L. Biagiotti and C. Melchiorri \\ DEIS, University of Bologna \\ Via Risorgimento 2, \\ 40136 Bologna, Italy \\ \{lbiagiotti, cmelchiorri\}@deis.unibo.it
}

\begin{abstract}
One of the main objectives of control algorithms for teleoperation systems is to have a master device mimicking the response of the remote environment, while the slave device is requested to behave as the human operator. In general, the remote environment is compliant, with a quite different behavior with respect to perfectly rigid surfaces (e.g. in surgery or human-centered applications). In these cases, the knowledge of the dynamical properties of the remote environment can be used in order to improve the transparency of the overall system. A number of analytical and computational models have been proposed in literature in order to describe the behavior of compliant materials but, for sake of simplicity, design and simulation of controllers for robotic telemanipulation are still tied to classical linear spring-damper models. On the other hand, previous experimental activities with soft materials and human tissues have demonstrated that they are characterized by dynamical effects (relaxation and creep phenomena), which cannot be taken into account by means of linear, low-order models. In this Chapter, we study the suitability of a class of nonlinear contact models to describe and emulate compliant visco-elastic environments. Their parameters, estimated on-line, can then be used to command a suitable behavior to the master device in order to render a better contact sensation to the user.
\end{abstract}

\section{Introduction}

On-line estimation of the environment dynamics plays an important role in the field of master/slave bilateral teleoperation systems, used by human operators to interact with remote environments. It is known [1] that the fidelity of force feedback, usually defined transparency, is affected by controllers and time delays in data transmission. This sensation is important e.g. in surgical applications, where the surgeon should be able to recognize human tissues by touching them and to identify their mechanical properties. Therefore, on-line estimation of mechanical properties of soft tissues can be used to (partially) recover transparency and telepresence sensation, as suggested by Colgate [2] and Buss [3], thus making force feedback surgical systems more effective.

The first step towards the implementation of on-line impedance estimation of objects interacting with a robotic device is the choice of suitable 
contact models [4], able to describe contact dynamics, i.e. the relation between contact forces and deformations of contacting bodies. Focusing on onedimensional contacts, in this chapter it is shown that linear models, commonly used to describe the interaction with stiff environments, cannot be applied to describe a unilateral contact with soft materials, where viscous effects are relevant. More generally, linear models are not suitable to emulate the behavior of compliant interfaces, when large deformations are imposed. For these reasons, more sophisticated nonlinear models, suitable for realtime computation and online estimation of the parameters, are introduced. In particular, the Hunt-Crossley model [5] and the Quasi-linear model [6,7] are considered.

This chapter is organized as follows: in Sec. 2 a general architecture for telemanipulation systems including an online impedance estimator is introduced. Then, Sec. 3 provides an overview on contact models, from the linear ones to Hunt-Crossley and quasi-linear models. Sec. 4 describes the techniques for on-line recursive estimation of the parameters of the proposed models. These algorithms have been applied to different materials in order to provide experimental validation (Sec. 5) to the theoretical considerations. Conclusions are reported in the final Sec. 6.

\section{Telemanipulation systems with environment impedance estimation}

Estimation of remote impedance has been introduced in different ways in bilateral telemanipulation schemes in order to improve their performance. For example, in [8] the estimation of the environment parameters (stiffness and damping coefficients) is used to achieve a good force tracking at the slave side. However, the main goal of control architectures involving the identification of the environment dynamics, is the achievement of "transparency" [9], that is an ideal kinesthetic coupling between the human operator and the remote environment. In other terms, the impedance perceived by the operator must be equal to the environmental impedance. This is a basic requirement of any ideal telemanipulation system, but, in particular, it is of great importance for medical/surgical applications, in which the possibility of perceiving the impedance(/stiffness) of the remote environment is a fundamental factor for the success of the task performed.

In order to enhance the transparency of the overall system, the typical solution consists in including the environment properties in the master controller (and, possibly, the human operator characteristics in the slave controller). This approach, called impedance reflection originally proposed by Hannaford in [10], is conceptually reported in the scheme of fig. 1, which represents the most general form of this approach, including the reflection of the environment impedance and of the operator impedance as well, and the exchange of both position and force information from master to slave and vice-versa. In 


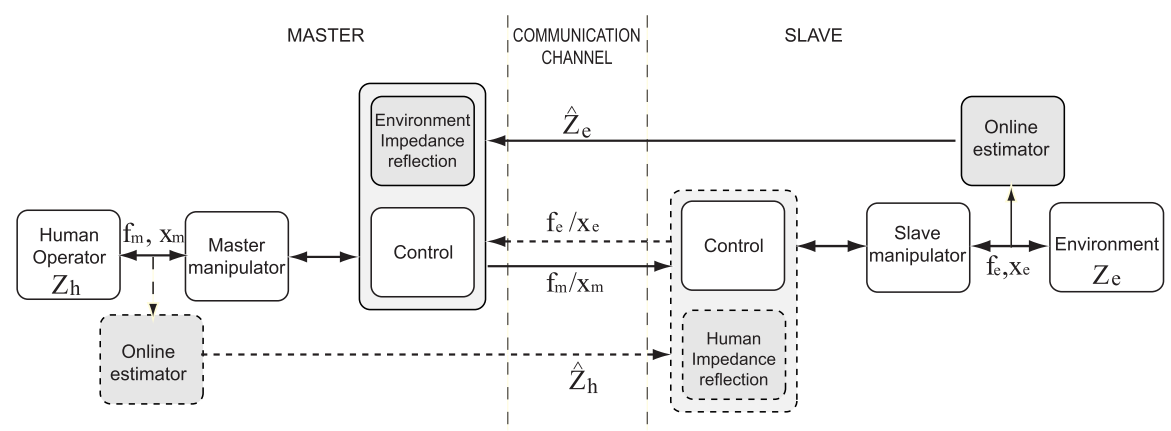

Fig. 1. Conceptual scheme of the impedance reflecting teleoperation.

many cases the teleoperation schemes are simplified versions of the general approach, in which, for example, only the remote impedance is reflected to the human operator to improve the perception of the environment and, in the other direction, only the master position is transmitted to the slave controller $[11,12]$. In other cases the impedance of the environment, see the impedance shaping telemanipulation architecture of Colgate [2], is applied to the operator with proper modifications which allow to obtain particular impression or perception, e.g. the kinematic similarity between the actual impedance and the apparent impedance in tasks with different scales.

In principle, the telemanipulation schemes with impedance reflection can be assimilated to haptic systems. In an haptic system the operator interacts, by means of the master devices, with a model-based virtual environment. For this reason, the models proposed in the next sections can be adopted, without any difference, in this field. In teleoperation, the user (partially or totally) interacts with an emulated environment, but in this case the model is built online, with the characteristic parameters of the contacted environment to be properly estimated. Therefore, a very crucial element in the overall system is the estimator, which must guarantee at the same time a fast convergence rate and the necessary approximation of the identified parameters to true values. For this reason, standard estimators are based on linear systems, whose algorithms are well-settled and computationally efficient. On the other hand, it is worth to notice that recently in teleoperation there is a strong interest towards the interaction with soft environments (e.g. for surgery and medical palpation) which involves large deformations and nonlinear phenomena. Therefore, in order to obtain very realistic responses, the linear contact models usually adopted should be revised and improved by considering nonlinear terms. At the same time, the identification of the parameters should remain feasible from a computational point of view. 


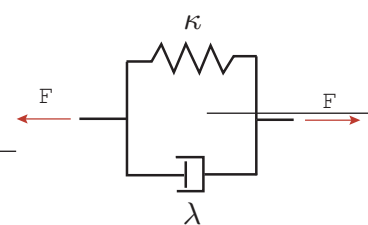

(a)

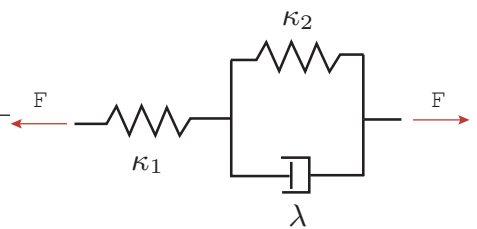

(b)

Fig. 2. Standard linear models: Kelvin-Voigt (a) and Zener (b).

\section{Contact models}

In the following, some contact models that can be used to describe the interaction between robot and environment are discussed. In particular, only one-dimensional models are described in order to present the main ideas and problems. Extension to multi-dimensional models is a current research activity.

\subsection{Linear contact models}

In many cases, within certain ranges of the involved variables, the linear theory is sufficient to describe the mechanical impedance of an object. In this case, a number of mechanical models, composed by linear springs and linear viscous dampers arranged in different configurations (e.g. Maxwell and Kelvin models), can be adopted. These models are widely used because of their simplicity and their clear physical interpretation [13], [4]. In this case, a general representation of the force-displacement relation is given by ordinary differential equations with constant coefficients, which depend on the model structure as well as on the considered material. The simplest example of linear model is the Kelvin-Voigt contact model (see fig. 2.a), composed by the mechanical parallel of a linear spring and a damper.

If $F$ is the force exerted by the material on a probe during contact, the linear model is expressed by:

$$
F(t)= \begin{cases}\kappa \delta(t)+\lambda \dot{\delta}(t) & \delta \geq 0 \\ 0 & \delta<0\end{cases}
$$

where $\dot{\delta}$ is the penetration velocity of the probe and $k$ and $\lambda$ are the elastic and viscous parameters of the contact. When the visco-elastic behavior of the material/object under analysis is remarkable, more complex models are available. A standard linear viscoelastic solid model (Zener model) is shown in fig. 2.b. Such a model is frequently adopted to represent behaviors in which instantaneous and delayed elasticities arise. Force and displacements 


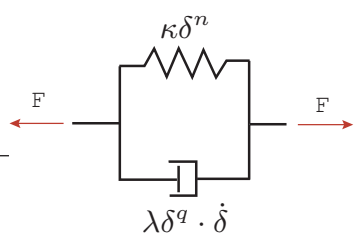

Fig. 3. Hunt-Crossley model.

are related by the following differential equation:

$$
F+\frac{\lambda}{\kappa_{1}+\kappa_{2}} \dot{F}=\frac{\kappa_{1}}{\kappa_{1}+\kappa_{2}} \cdot\left(\kappa_{2} \delta+\lambda \dot{\delta}\right) \quad \delta \geq 0
$$

where the meaning of the parameters $\kappa_{1}, \kappa_{2}$ and $\lambda$ is shown in fig. 2.b.

\section{$3.2 \quad$ Nonlinear contact models}

\section{Lumped parameters systems with non linear coefficient: the Hunt-} Crossley case

When the linear hypothesis is not applicable, non linear models must be considered. For the sake of simplicity, many authors developed dynamic models of contacting systems drawing inspiration from classical linear models and introducing nonlinearities in the definition of the spring stiffness and of the dashpot viscosities $[14,15]$. A noteworthy nonlinear model has been proposed by Hunt and Crossley [16] and adopted by several authors $[17,18,19]$ to describe the dynamic behavior of the interface between a robot and the environment when a contact occurs. It consists of a Kelvin model, eq. (1), where the stiffness of the spring and the viscosity of the damper are expressed by a power-law of the displacement. The differential form of this model is:

$$
F(t)=\kappa \delta^{n}+\lambda \delta^{q} \cdot \dot{\delta}
$$

where usually it is assumed $q=n$, with the exponent $n \in \mathbb{R}$ usually close to the unity, that takes into account the geometry of contact surfaces. Notice that when $n=3 / 2$ the elastic term of (3) exactly matches the force resulting from the Hertzian theory for spheres contacting in static conditions [6].

\section{An integral form for nonlinear systems: the quasi-linear model}

Models like that of Hunt and Crossley are based on constitutive equations expressed in differential form. A different approach consists of considering directly the force response due to a change step in displacement, which is expressed by the relaxation function

$$
F_{0}(t)=\Psi\left(\delta_{0}, t\right)
$$


and superimposing each contribution of a displacement history by applying a suitable superposition principle. In order to simplify the treatment, following the hypothesis originally formulated by Fung [20], the relaxation function is assumed to be of the form:

$$
\Psi(\delta, t)=F^{(e)}(\delta) \cdot g(t) \quad \text { with } \quad g(0)=1
$$

where $F^{(e)}(\delta)$ is the elastic response, that is the amplitude of the force generated instantaneously by a displacement $\delta$, while $g(t)$ is the reduced relaxation function, describing the time-dependant behavior of the material.

In order to find the force response to a generic displacement $\delta(t)$, one can model the input function as a sequence of steps. The force produced by an infinitesimal displacement $d \delta(\tau)$, superposed in a state of displacement $\delta$ at an instant of time $\tau$ is, for $t>\tau$ :

$$
d F(t)=\frac{\partial \Psi[\delta(\tau), t-\tau]}{\partial \delta} d \delta(\tau)
$$

and, considering the Fung's hypothesis, expressed in (5), one obtains

$$
d F(t)=g(t-\tau) \frac{d F^{(e)}[\delta(\tau)]}{d \delta} d \delta(\tau)
$$

By applying a modified superposition principle, discussed in $[7,21,22]$, the total force at the instant $t$ is the sum of the contribution of all the past changes, i.e.

$$
F(t)=\int_{-\infty}^{t} g(t-\tau) \frac{d F^{(e)}[\delta(\tau)]}{d \delta} \frac{d \delta(\tau)}{d \tau} d \tau
$$

That equation can be rewritten as

$$
F(t)=\int_{0}^{t} g(t-\tau) K^{(e)}[\delta(\tau)] \dot{\delta}(\tau) d \tau
$$

where the lower limit of the integral has been changed assuming that the motion starts at time $t=0$ and $F^{(e)}=0, \delta=0$ for $t<0$. The term $K^{(e)}(\delta)=\frac{d F^{(e)}[\delta]}{d \delta}$ is the elastic stiffness and $\dot{\delta}(\tau)$ is the rate of displacement.

The expressions of the reduced relaxation function and of the elastic response in (9) determine the model of the contact. According to the Fung's hypotheses, expressed by equation (5), the relaxation function $g(t)$ is a decreasing, continuous function of the time, normalized to 1 at $t=0$. It is usually assumed to be composed by a linear combination (with the coefficient $c_{i}$ depending on the material) of exponential functions

$$
g(t)=\sum_{i=0}^{r} c_{i} e^{-\nu_{i} \cdot t} \quad \text { with } \quad \sum_{i=0}^{r} c_{i}=1
$$


whose exponents $\nu_{i}$ identify the rate of the relaxation phenomena. The number $r$ and the value of such parameters depend on the behavior of the system under analysis, while $\nu_{0}=0$. From this definition of the reduced relaxation function $g(t)$ it descends that $F^{(e)}(\delta)$ can be approximated by the force response in a loading experiment with a sufficiently high rate of displacement.

The nonlinear elastic response can be modelled by different analytical expressions. Two of the most significant models adopted in the literature are:

$$
\begin{aligned}
& K^{(e)}(\delta)=m \cdot e^{b \delta} \\
& K^{(e)}(\delta)=p \cdot \delta^{q}
\end{aligned}
$$

where $(m, b)$ and $(p, q)$ are parameters dependent on the material and the geometry taken into account. The expression of $F^{(e)}(\delta)$ descends directly from the integration of equation (11) or (12), with respect to $\delta$ :

$$
\begin{aligned}
& F^{(e)}(\delta)=\frac{m}{b}\left(e^{b \delta}-1\right) \\
& F^{(e)}(\delta)=\frac{p}{q+1} \delta^{q+1}
\end{aligned}
$$

The quasi-linear model has a simple and meaningful interpretation. Equation (9) describes in the time domain the signal $K^{(e)}(\delta) \dot{\delta}$ filtered by a linear system represented by its transfer function:

$$
G(s)=\mathcal{L}\{g(t)\}=\frac{c_{0}}{s}+\frac{c_{1}}{s+\nu_{1}}+\ldots+\frac{c_{i}}{s+\nu_{i}}+\ldots+\frac{c_{r}}{s+\nu_{r}}
$$

The number $r$ of characteristic modes of the filter $G_{F}(s)$ should be carefully chosen on the basis of the viscoelasticity of the surface in contact. Strongly viscoelastic materials require a number $r$ quite large, while quasi-elastic materials can be modelled by assuming $r=1$. The input signal of the linear filter can be rewritten as:

$$
K^{(e)}(\delta) \dot{\delta}=\frac{d F^{(e)}(\delta)}{d \delta} \dot{\delta}=\frac{d F^{(e)}(\delta(t))}{d t}
$$

Being the derivative a linear operator, from (9) and (16) it comes out that it exists a linear relation between the elastic response $F^{(e)}(\delta)$ and the force produced by the system. Such a relationship is given by the transfer function

$$
\begin{aligned}
G_{L}(s) & =s\left(\frac{c_{0}}{s}+\frac{c_{1}}{s+\nu_{1}}+\frac{c_{2}}{s+\nu_{2}}+\ldots+\frac{c_{r}}{s+\nu_{r}}\right) \\
& =\frac{\left(\sum_{i=0}^{r} c_{i}\right) s^{r}+\ldots+c_{0}\left(\prod_{i=0}^{r} \nu_{i}\right)}{s^{r}+\ldots+\left(\prod_{i=0}^{r} \nu_{i}\right)}
\end{aligned}
$$

where, for both the numerator and the denominator, only the coefficients of terms of maximum and minimum degree are explicitly reported. It is worth 


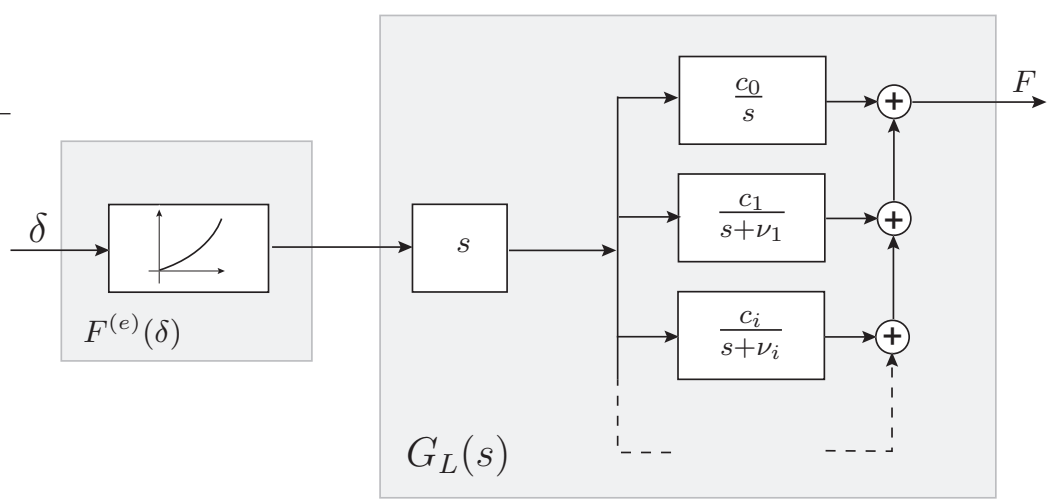

Fig. 4. Block diagram of the quasi-linear model: static nonlinear part and dynamic linear function.

to notice that the relative degree of the function $G_{L}(s)$ is zero. Therefore, when a step (of amplitude $\widehat{F^{e}}$ ) is applied to the system, its response will be discontinuous and will start from $\widehat{F^{e}}$ (note that in (17) $\sum_{i=0}^{r} c_{i}=1$ ).

In Fig. 4 the complete model of a viscoelastic material is reported; it is composed by two elements, connected in cascade:

- a nonlinear static block which has the displacement $\delta$ as input and provides the instantaneous elastic response $F^{(e)}$;

- a linear dynamic block $G_{L}(s)$, which takes into account the (typically slow) dynamic behavior of the material.

Such a model is usually referred to as Hammerstein model.

\section{Procedures for online estimation}

\subsection{Identification procedure for linear models}

As an example we consider the Zener model, whose differential equation corresponds to the discrete-time representation ${ }^{1}$ :

$$
F(h)=-\left[\frac{\kappa_{1}+\kappa_{2}}{\lambda} T-1\right] F(h-1)+\kappa_{1} \delta(h)+\left[\frac{\kappa_{1} \kappa_{2}}{\lambda} T-\kappa_{1}\right] \delta(h-1)
$$

where $F(h)$ and $\delta(h)$ are the samples of the force $F(t)$ and of the displacement $\delta(t)$ at the instants $t_{h}=h T$, being $T$ the sampling time.

Equation. (18) is a classical example of auto-regressive (ARX) model,

$$
y(h)=\theta^{T} \varphi(h)
$$

${ }^{1}$ The Euler approximation $\frac{d y(t)}{d t} \approx \frac{y(h)-y(h-1)}{T}$ has been adopted in this case. 
where $y(h)=F(h), \varphi(h)=\left[\begin{array}{lll}-F(h-1) \delta(h) \delta(h-1)\end{array}\right]^{T}$ and $\theta=\left[\begin{array}{lll}\theta_{1} & \theta_{2} & \theta_{3}\end{array}\right]^{T}=$ $\left[\begin{array}{lll}\left(\frac{\kappa_{1}+\kappa_{2}}{\lambda} T-1\right) & \kappa_{1} & \left(\frac{\kappa_{1} \kappa_{2}}{\lambda} T-\kappa_{1}\right)\end{array}\right]^{T}$. If one assumes a model of this kind, the on-line estimation of the unknown parameters $\hat{\theta}$ is straightforward. A standard recursive algorithm [23], derived from classical least-square methods employed for off-line identification, can be adopted with the aim of minimizing the cost function:

$$
\begin{aligned}
& V_{N}(\theta):=\frac{1}{N} \sum_{h=1}^{N} \alpha_{h} \varepsilon^{2}(h) \\
& \varepsilon(h):=y(h)-\theta^{T} \varphi(h)
\end{aligned}
$$

where $N$ is the number of measures acquired from the beginning of the estimation process, and $\alpha_{h}$ are proper weights. In particular, since the aim is to obtain an estimation of possibly time-varying parameters, it has been assumed

$$
\alpha_{h}=\beta^{N-h}
$$

where $\beta<1$ is the forgetting factor. With this expression of the weights, it is clear that older samples have less importance than recent ones. The expression of the recursive estimators is:

$$
\begin{aligned}
& \hat{\theta}(h)=\hat{\theta}(h-1)+Q(h)\left[y(h)-\varphi(h)^{T} \hat{\theta}(h-1)\right] \\
& Q(h)=P(h-1) \varphi(h)\left[\beta+\varphi(h)^{T} P(h-1) \varphi(h)\right]^{-1} \\
& P(h)=\frac{1}{\beta}[\mathbb{I}-Q(h)] P(h-1)
\end{aligned}
$$

At each sampling time, the recursive least square algorithm $(R L S)$ computes new values of the unknowns and update its status on the basis of new data, but to start the estimation process the initial conditions on $\hat{\theta}(0)$ and $P(0)$ are necessary. A common choice is to take $P(0)=c \cdot I$, where $c$ is a proper constant scalar, and $\hat{\theta}(0)=0$, but it can see that the importance of these initial values decays with the time, as the number of new data grows. Also for this reason, a critical point in the definition of the estimator is the choice of the forgetting factor $\beta$, which must guarantee a proper tradeoff between tracking ability and noise sensitivity. The need to perceive variations in the touched materials suggests to compute the forgetting factor $\beta$ dynamically, according to the magnitude of the error $|F(h)-F(h)|$ between the estimated and the measured force [19]. In particular, when the error is small, the forgetting factor should be close to one, while it has to be decreased for large errors, so that the weight related to older samples decays. In particular, as reported in fig. $5, \beta$ is computed as

$$
\beta=1-\alpha_{1}\left(\frac{1}{\pi} \arctan \left(\alpha_{2}|F(h)-\hat{F}(h)|-\alpha_{3}\right)+\frac{1}{2}\right)
$$




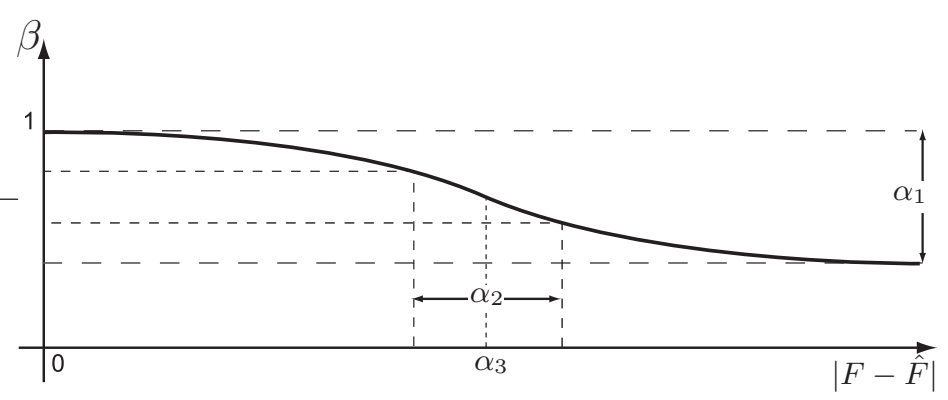

Fig. 5. Forgetting factor adaptation depending on error. Parameters $\alpha_{1}, \alpha_{2}$, and $\alpha_{3}$ must be adjusted on the basis of the noise level.

where $1-\alpha_{1}$ is the forgetting factor value for large errors, $\alpha_{3}$ has the meaning of a threshold between the small error and the large error condition, while the amplitude of the transition region is governed by $\alpha_{2}$.

\subsection{Identification method for Hunt-Crossley parameters}

Also in the case of the Hunt-Crossley model, a linear regression algorithm is applied. Since (3) is non linear with respect to the exponent $n$, the main idea is to separate the estimation of $k$ and $\lambda$ from the estimation of $n$. In this way, we can write two recursive least-squares estimators $\Gamma_{1}$ and $\Gamma_{2}$ [23] interconnected via feedback, as shown in Fig. 6.

In particular, $\Gamma_{1}$ estimates $k$ and $\lambda$ minimizing the cost function $V_{N}(k, \lambda)$, which has the same structure of (20) with the error defined as follows:

$$
\varepsilon_{1}(h):=F(h)-[k+\lambda \dot{\delta}(h)] \delta^{n}(h)
$$

Therefore, by assuming the vector of estimates at time $t=h T \hat{\theta}_{1}(h)=$ $[\hat{k}(h), \hat{\lambda}(h)]^{T}$, the vector of input signals $\varphi_{1}(h)=\left[\delta^{n}(h), \delta^{n}(h) \dot{\delta}(h)\right]^{T}$ and the system output $y_{1}(h)=F(h)$, the estimator $\Gamma_{1}$ is implemented in the standard recursive form (23), already used for linear models.

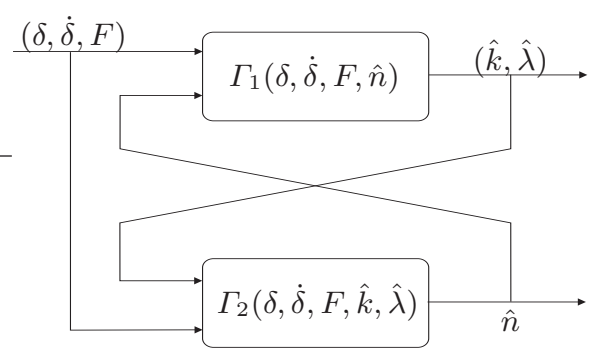

Fig. 6. On-line parameters estimator for the Hunt-Crossley model 
On the other side, an expression of (25) that is linear with respect to the parameter $n$ can be obtained as

$$
\ln \varepsilon_{2}(h)=\ln \frac{F(h)}{k+\lambda \dot{\delta}(h)}-n \ln \delta(t)
$$

with

$$
\varepsilon_{2}(h):=1+\frac{\varepsilon_{1}(h)}{[k+\lambda \dot{\delta}(h)] \delta^{n}(h)}
$$

If $\varepsilon_{1}$ is small with respect to the force computed according to the HuntCrossley model (3), it is possible to write the following series expansion of (26):

$$
\ln \varepsilon_{2}=\ln \left(1+\frac{\varepsilon_{1}}{[k+\lambda \dot{\delta}] \delta^{n}}\right) \simeq \frac{\varepsilon_{1}}{[k+\lambda \dot{\delta}] \delta^{n}}
$$

Therefore, if the previous assumption holds and $\left\langle\varepsilon_{1}\right\rangle$ is independent on the force computed according to $(3),\left\langle\ln \left(\varepsilon_{2}(h)\right)\right\rangle$ can be considered as a stochastic process with zero mean and it is possible to estimate $n$ by means of a recursive least-square procedure minimizing the cost function $W_{N}(n)$ :

$$
W_{N}(n):=\frac{1}{N} \sum_{h=1}^{N} \ln ^{2}\left(\varepsilon_{2}(h)\right)
$$

and the implementation of the estimator $\Gamma_{2}$ is again analogous to (23), with $\hat{\theta}_{2}(h)=\hat{n}(h), \varphi_{2}(h)=\ln \delta(h)$ and $y_{2}(h)=\ln (F(h) /(k+\lambda \dot{\delta}(h)))$.

Because of the feedback interconnection (see fig. 6), the value of $n$ used by $\Gamma_{1}$ as well as the value of these parameters used by $\Gamma_{2}$ is not the "true" value but the estimated one. For this reason, beside measurement noise and model error, each estimator behaves as an additional source of noise that could compromise the convergence of the algorithm. These considerations lead to rewrite estimation errors (25) and (26) as:

$$
\begin{aligned}
& \varepsilon_{1}^{\prime}(h)=F(h)-(k+\lambda \dot{\delta}(h)) \delta^{\hat{n}}(h) \\
& \ln \varepsilon_{2}^{\prime}(h)=\ln \frac{F(h)}{\hat{k}+\hat{\lambda} \dot{\delta}(h)}-n \ln \delta(h)
\end{aligned}
$$

where the effect introduced by the use of estimates instead of true parameters values is considered. Therefore, provided that elementary estimators would converge, the convergence of their feedback interconnection is obtained if additional disturbances do not bias the stochastic processes $\left\langle\varepsilon_{1}^{\prime}(h)\right\rangle,\left\langle\ln \left(\varepsilon_{2}^{\prime}(h)\right)\right\rangle$ expressing residuals between measured and estimated forces for $(\hat{k}, \hat{\lambda})$ and $\hat{n}$ respectively. 
In particular, (30) can be rewritten as:

$$
\varepsilon_{1}^{\prime}(h)=\varepsilon_{1}(h)+(k+\lambda \dot{\delta}(h)) \delta^{n}(h)\left(1-\delta^{\delta n}\right)
$$

and, if the estimation error $\delta n$ is small, the following approximation holds:

$$
1-\delta^{\delta n} \simeq \delta n \ln \delta(h)
$$

Therefore, $\left\langle\varepsilon_{1}^{\prime}(h)\right\rangle$ is a zero mean stochastic process if:

$$
E[\delta n] E\left[(k+\lambda \dot{\delta}(h)) \delta^{n}(h)\right]=0
$$

and this condition is satisfied if the estimator $\Gamma_{2}$ converges independently on $\Gamma_{1}$.

Since the relation between $\varepsilon_{2}$ and $\varepsilon_{2}^{\prime}$ is given by:

$$
\begin{aligned}
\ln \varepsilon_{2}^{\prime}(h) & =\ln \varepsilon_{2}(h)-\ln \frac{\hat{k}+\hat{\lambda} \dot{\delta}(h)}{k+\lambda \dot{\delta}(h)} \\
& =\ln \varepsilon_{2}(h)-\ln \left(1+\frac{\delta k+\delta \lambda \delta(\dot{h})}{k+\lambda \delta(\dot{h})}\right)
\end{aligned}
$$

and by computing the series expansion, also recalling (28), we obtain:

$$
\ln \varepsilon_{2}^{\prime} \simeq \ln \varepsilon_{2}-\frac{\delta k+\delta \lambda \dot{\delta}}{k+\lambda \dot{\delta}} \simeq \frac{\varepsilon_{1}-\delta^{n}(\delta k+\delta \lambda \dot{\delta})}{\delta^{n}(k+\lambda \dot{\delta})}
$$

Hence, the additional noise due to feedback interconnection has to be dominated by $\varepsilon_{1}(h)$ so that $\left\langle\ln \left(\varepsilon_{2}^{\prime}(h)\right\rangle\right.$ is still a zero mean process:

$$
\|\delta k+\delta \lambda \dot{\delta}\| \ll\left\|\frac{\varepsilon_{1}}{\delta^{n}}\right\|
$$

This condition is not too restrictive, because at the beginning of the estimation process, when the estimation errors $\delta k$ and $\delta \lambda$ can be considerable, the penetration $\delta$ is small. Moreover $\dot{\delta}$ is also small, since the Hunt-Crossley model is valid for a limited range of impact velocities. Therefore, the use of $(\hat{k}, \hat{\lambda})$ within the $\Gamma_{2}$ estimator does not alter its convergence properties and the overall feedback estimator provides unbiased estimates $\hat{k}, \hat{\lambda}, \hat{n}$ of parameters of the Hunt-Crossley model.

\subsection{Estimation of quasi-linear model parameters}

In order to estimate the characteristic parameters of the quasi-linear model it is necessary to have a sampled-data system. This can be obtained by simply using a digital filter in lieu of $G_{L}(s)$ (see fig. 4), whose general form is

$$
G_{L}\left(z^{-1}\right)=z^{-k} \frac{b_{0}+b_{1} z^{-1}+\ldots+b_{l} z^{-l}}{1+a_{1} z^{-1}+\ldots+a_{r} z^{-r}}
$$




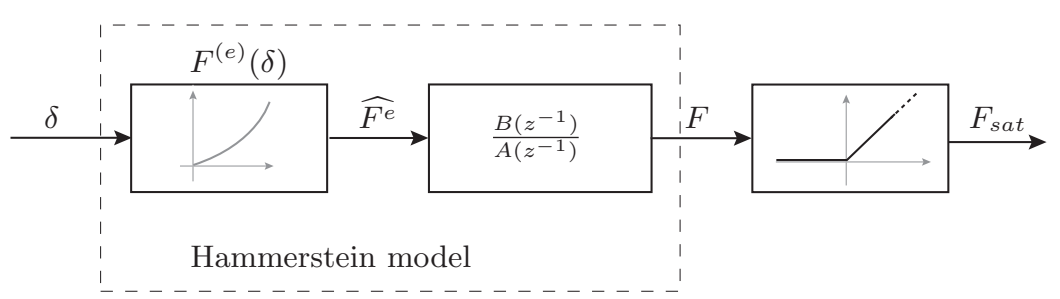

Fig. 7. Hammerstein model with saturated output of a contact with a viscoelastic material.

Therefore, from the scheme of Fig. 4 the model reported in Fig. 7 is obtained, where the continuous transfer function $G_{L}(s)$ has been replaced by the digital filter $G_{L}\left(z^{-1}\right)^{2}$. Note that, from the considerations reported in sec. 3.2, it follows that:

- no pure delay exists in the system between the input (of the linear systems) $\widehat{F}^{e}$ and the output $F(k=0)$;

- the response depends on the input at the same instant; therefore the orders of the numerator of $G_{L}\left(z^{-1}\right)$ and that of the denominator must be equal $(l=r)$.

Finally, it is worth to notice that in the general case the model, composed by a cascade of a nonlinear and of a linear part, includes a redundancy in the parameters definition. As a matter of fact, the gain of overall system results from the product of the gain of nonlinear characteristic and of that of the linear part. In our case, this ambiguity has been solved by assuming that the coefficient $b_{0}$ in (38) is equal to 1 . In this way, the initial value of $F$ to a unit step input

$$
F\left(0^{+}\right)=\lim _{z \rightarrow+\infty} G_{L}\left(z^{-1}\right) \frac{1}{1-z^{-1}}=b_{0}=1
$$

is the same of that produced by $G_{L}(s)$

$$
\lim _{s \rightarrow+\infty} s\left(G_{L}(s) \frac{1}{s}\right)=\lim _{s \rightarrow+\infty} s G(s)=g\left(0^{+}\right)=1
$$

and, accordingly, the (physical) meaning of the elastic response $F^{(e)}$ remain unchanged.

The scheme of Fig. 7 reproduces the well-known Hammerstein model, frequently adopted to model and identify nonlinear systems [24]. This structure is quite interesting, since it is composed by a no-memory nonlinear gain and a dynamic linear block, resulting particularly suitable for identification $[25,26]$.

\footnotetext{
${ }^{2}$ The saturation on the model output avoids that the force response becomes negative, but does not interfere with the identification process.
} 


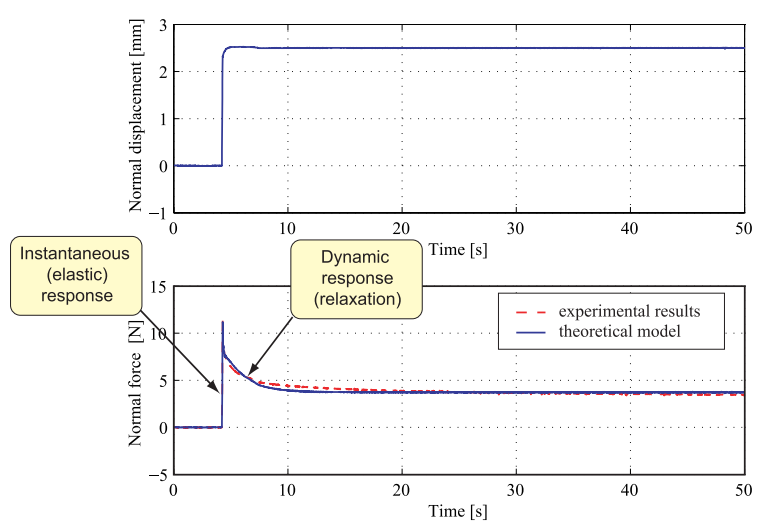

Fig. 8. Dynamic response of polyurethane gel (dashed) and estimated model (solid).

In particular, by exploiting the model structure with the two temporally distinct elements (the static part - elastic response $F^{(e)}$ - produces an instantaneous response, while the dynamic one - reduced relaxation function - reacts with a slower dynamic and in the first time instants does not influence the elastic response, see fig. 9), which reflects the typical behavior of soft materials during an interaction, it is possible to conceive a two phases estimation algorithm, see fig. 9:

- during the contact, provided that the velocity of the probe is fast enough (in principle an ideal step deformation should be used), the parameters of $F^{(e)}$ are estimated, by neglecting the dynamic terms;

- by using the output of the elastic response, the coefficients of the linear filter can be identified.

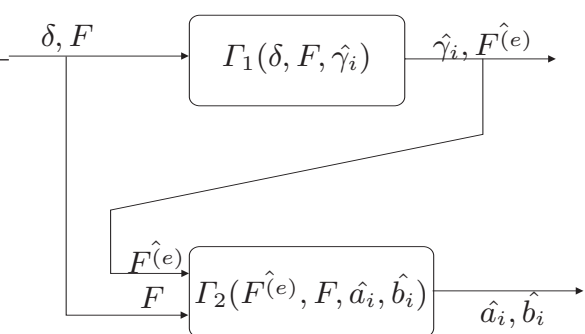

Fig. 9. Online parameters estimator for the Hammerstein model. 
For the elastic part, the expression based on the power law is the most convenient $^{3}$

$$
F^{(e)}(\delta)=\gamma_{1} \delta^{\gamma_{2}}
$$

since it can be easily transformed in a linear form, like in the case of HuntCrossley model:

$$
\ln \left(F^{(e)}\right)=\ln \left(\gamma_{1}\right)+\gamma_{2} \ln (\delta)
$$

Therefore, the estimation of parameters $\gamma_{i}$, can proceed by means of the $R L S$ algorithm as in $(23)$, with $\hat{\theta}_{1}(h)=\left[\ln \left(\hat{\gamma_{1}}(h)\right), \hat{\gamma_{2}}(h)\right]^{T}, \varphi_{1}(h)=[1, \ln (\delta(h))]^{T}$ and $y_{1}(h)=\ln (F(h))$. In particular, to compensate for the fact that the minimization is performed with respect to the norm of the squared error of $\ln \left(F^{(e)}\right)$ and not of $F^{(e)}$, a weighted least-square algorithm is adopted [27], with the weights chosen as

$$
\alpha_{h}=\beta^{N-h} e^{y_{1}(h)}
$$

In this case, the recursive estimation algorithm becomes:

$$
\begin{aligned}
\hat{\theta_{1}}(h) & =\hat{\theta_{1}}(h-1)+Q(h)\left[y_{1}(h)-\varphi_{1}(h)^{T} \hat{\theta_{1}}(h-1)\right] \\
Q(h) & =P(h-1) \varphi_{1}(h)\left[\left(\frac{\beta}{e^{y_{1}(h)}}\right)+\varphi_{1}(h)^{T} P(h-1) \varphi_{1}(h)\right]^{-1} \\
P(h) & =\frac{1}{\beta}[\mathbb{I}-Q(h)] P(h-1)
\end{aligned}
$$

It is worth to notice that only the data, which are acquired when the speed of the contacting probe (and therefore the speed of the deformation $\dot{\delta}$ ) is bigger than a given threshold $\dot{\delta}^{\star}$ (and $F \approx F^{(e)}$ ) [28]. Otherwise, the estimate $\theta_{1}$ is not updated, and only the estimation of the linear filter parameters $\left[\hat{a}_{i}, \hat{b_{i}}\right]$ is performed by $\Gamma_{2}$. In this case, the standard $R L S$ method is applied, being the linear filter a classical $A R X$ system, with the input $h a t F^{(e)}(h)$ and the output $F(h)^{4}$.

Note, that in this case the two estimators work in a cascade configuration (see fig. 9), and convergence problems do not arise. The only interaction is that the the input signal of the linear system is computed at each iteration on the basis of the the updated values of $\gamma_{i}$.

\section{Experimental results}

In order to verify the above estimation algorithm, a laboratory setup has been implemented. The setup consists of a linear electric motor equipped with a position sensor and a load cell, Fig. 10.

\footnotetext{
${ }^{3}$ Compare with (14), where $\gamma_{1}=\frac{p}{q+1}, \gamma_{2}=q+1$.

${ }^{4}$ Being the parameter $b_{0}=1$ the estimation (23) for the linear filter is performed with $\hat{\theta}_{2}(h)=\left[-\hat{a}_{1},-\hat{a}_{2}, \ldots,-\hat{a}_{r}, \hat{b}_{1}, \hat{b}_{2}, \ldots, \hat{b}_{r}\right]^{T}, \varphi_{2}(h)=[F(h-1), F(h-$ $\left.2), \ldots, F(h-r), \hat{F^{(e)}}(h-1), \hat{F^{(e)}}(h-2), \ldots, \hat{F^{(e)}}(h-r)\right]^{T}$ and $y_{2}(h)=F(h)-$ $F^{(e)}(h)$.
} 


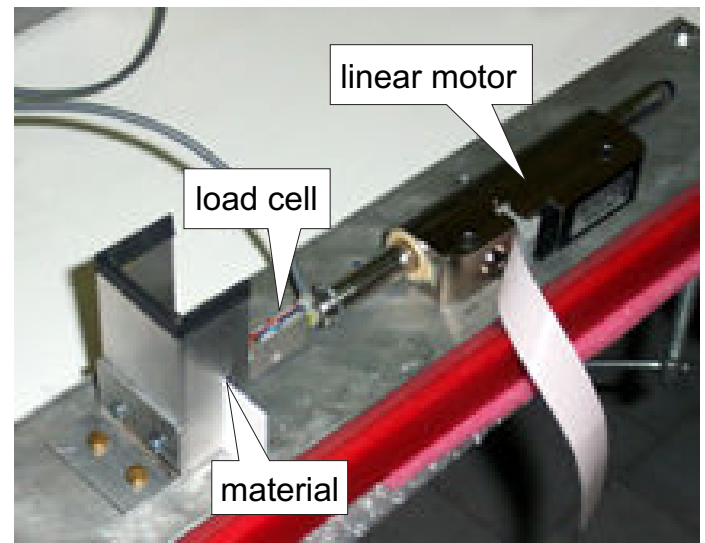

Fig. 10. Experimental setup.

The measures required by the estimation algorithm have been obtained by imposing a motion profile to a linear motor in contact with different materials. The contact force $F$ is measured by means of the load cell, the penetration $\delta$ is measured by comparing the current motor position with the position measured at the time of impact, and finally the penetration velocity $\dot{\delta}$ is obtained from $x$ by means of a state variable filter. The linear motor and the load cell have been connected, by means of a AD/DA board, to a standard PC running control and estimation algorithms in a mixed MATLAB/RTLinux environment. In the experimental activity, the sampling time has been set to $T=1$ msec. Several materials have been used and here, in particular, the results obtained with a thin layer of plastic material, characterized by a stiff behavior, and a soft gel, whose viscoelasticity is relevant, are described.

\subsection{Hunt-Crossley parameters estimation}

\section{Stiff material}

Experimental results related to the thin layer of plastic materials are presented in Fig. 11 and in Fig. 12. In particular, the hysteresis loop reported in Fig. 11(a) shows that energy dissipation is low and the behavior of the material depends essentially on the elastic coefficient $k$. Moreover, hysteresis loop and the related power exchange, reported in Fig. 11(b), show good correspondence between the experimental curves and the estimated one. Finally, Fig. 12 shows the parameters estimates. In particular, the following values are obtained: $k \simeq 1.15 e 4, \lambda \simeq 70, n \simeq 1.2$. According to what previously discussed about properties of the Hunt-Crossley model, the exponent $n$, that takes into account geometry of contact surfaces, is about one. We notice that $k$ and $n$ are quite stable around their final value, while $\lambda$ presents a more 


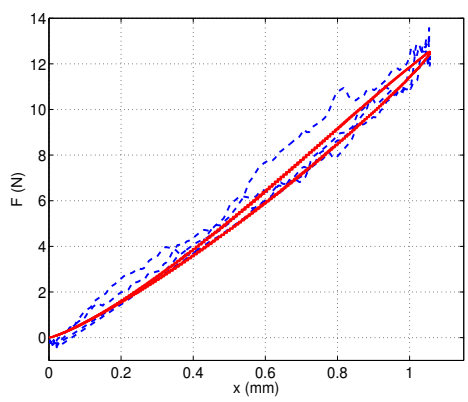

(a) Hysteresis loop

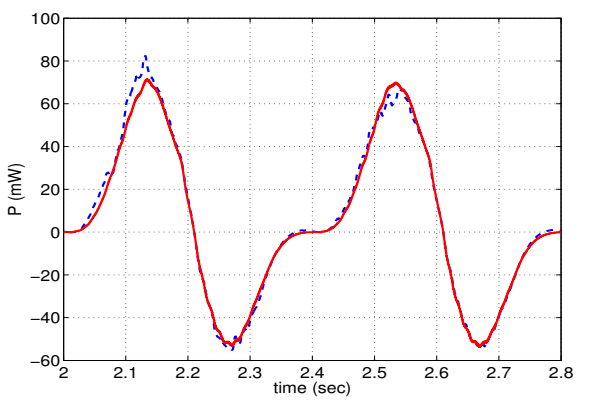

(b) Power Exchange

Fig. 11. Hunt-Crossley model: measured (dashed) and estimated (solid) hysteresis loop and power exchange for a thin layer of plastic materials

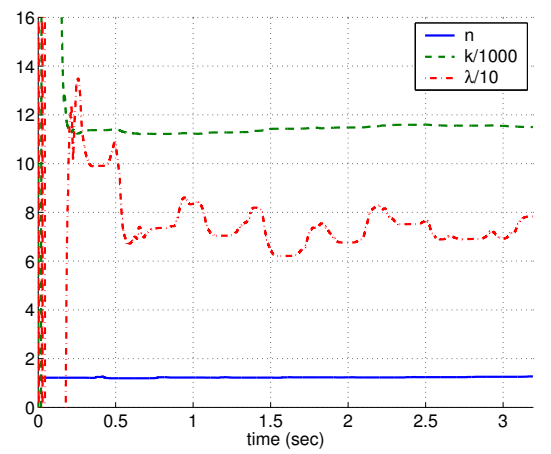

Fig. 12. Hunt-Crossley model: estimation of $n$ (solid), $k$ (dash) and $\lambda$ (dash-dot) for a thin layer of plastic material

oscillatory behavior, because of numerical differentiation used to compute $\dot{x}$ which makes this parameter more sensitive to measurement noise.

\section{Soft material}

For compliant materials, the advantages of the Hunt-Crossley model are more evident. The application of a linear regression algorithm to estimate parameters $K$ and $B$ of the Kelvin-Voigt model for a soft gel provides the results of Fig. 13, with $K \simeq 2.26 e 3 \mathrm{~N} / \mathrm{m}$ and $B \simeq 123 \mathrm{Ns} / \mathrm{m}$. In particular, drawbacks related to nonzero estimated force when $x=0$ are evident, as well as inconsistencies in power exchange between the probe device and the soft gel. On the contrary, hysteresis loop estimated by means of the Hunt-Crossley model is more similar to the measured one (Fig. 14(a)), as well the estimated 


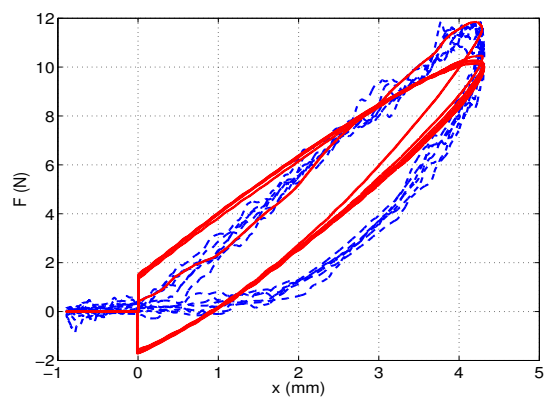

(a) Hysteresis loop

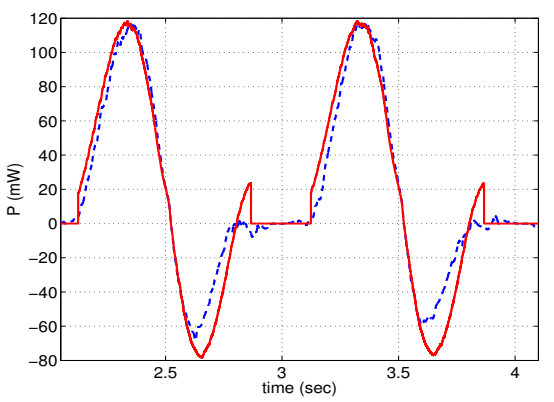

(b) Power Exchange

Fig. 13. Linear model: measured (dash) and estimated (solid) hysteresis loop and power exchange for soft gel

power exchange, shown in Fig. 14(b), that does not exhibit positive "spikes" present in Fig. 13(b).

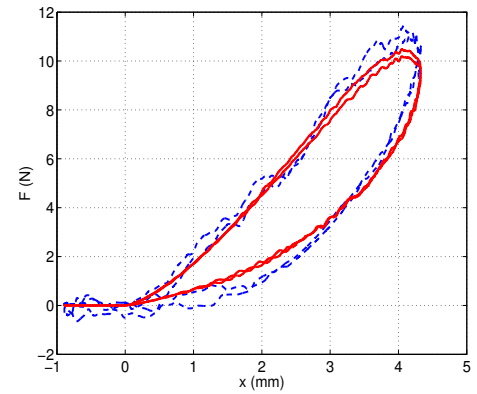

(a) Hysteresis loop

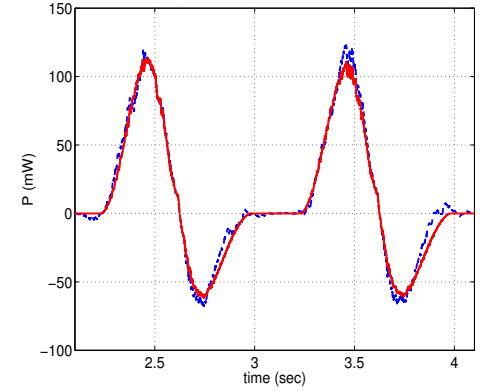

(b) Power Exchange

Fig. 14. Hunt-Crossley model: measured (dashed) and estimated (solid) hysteresis loop and power exchange for soft gel

Finally, Fig. 15 shows the convergence of parameters to their final values $k \simeq 1.35 e 3, \lambda \simeq 36, n \simeq 1.35$. Notice that in this case the value of $n$ is greater than that obtained for the stiff material, since contact surface between the probe device and the soft gel is slightly different.

\section{Change of material}

As mentioned in Sec. 4, the use of a forgetting factor allows to improve the detection of material changes. In particular, the case of a switching from the soft gel to the layer of plastic materials has been considered. The hysteresis 


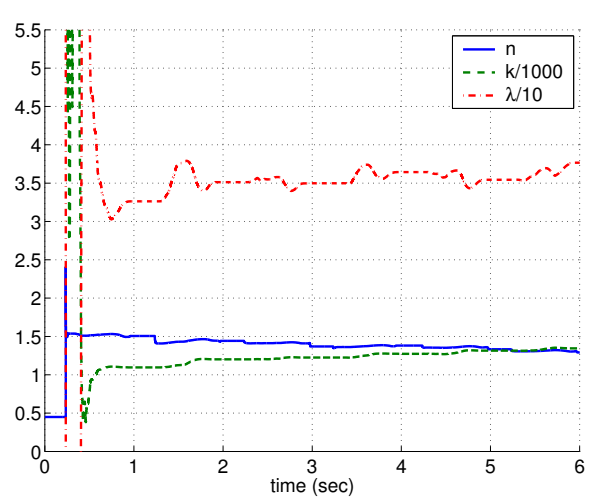

Fig. 15. Hunt-Crossley model: estimation of $n$ (solid), $k$ (dash) and $\lambda$ (dash-dot) for soft gel

loop and the power exchange diagram are shown in Fig. 16, which confirm the adequacy of the Hunt-Crossley model to describe both stiff and soft materials. The ability of the estimation algorithm to detect a change in the touched

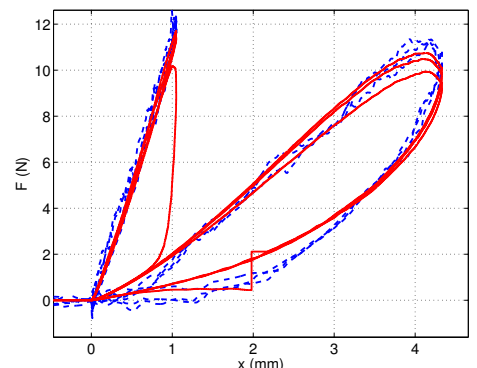

(a) Hysteresis loop

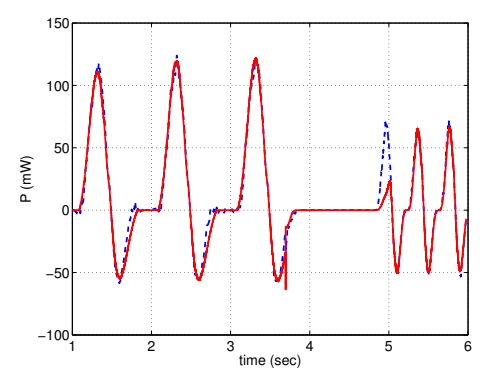

(b) Power Exchange

Fig. 16. Hunt-Crossley model: measured (dashed) and estimated (solid) hysteresis loop and power exchange when soft gel is substituted by the thin plastic layer

material is shown in Fig. 17. Indeed, after a short transient, the convergence to parameters of the new material is achieved and previous estimates do not affect final values.

\subsection{Hammerstein model estimation}

The Hammerstein model is suitable to describe the interaction with both elastic and viscoelastic materials. In particular, it is quite convenient for modelling the behavior of those objects which are characterized by a very 


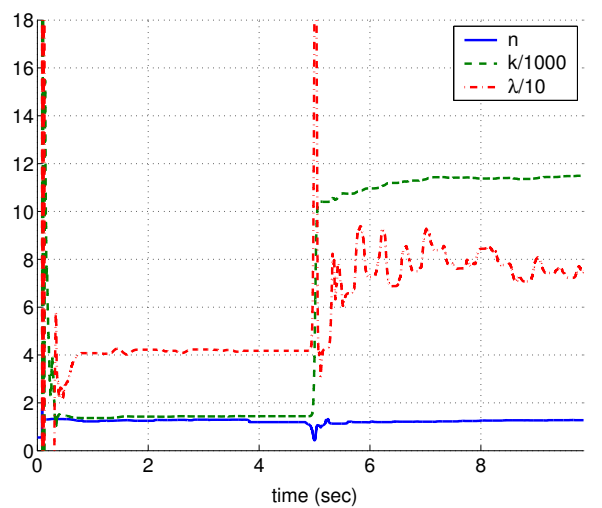

Fig. 17. Hunt-Crossley model: estimation of $n$ (solid), $k$ (dash) and $\lambda$ (dash-dot) when soft gel is substituted by the thin plastic layer
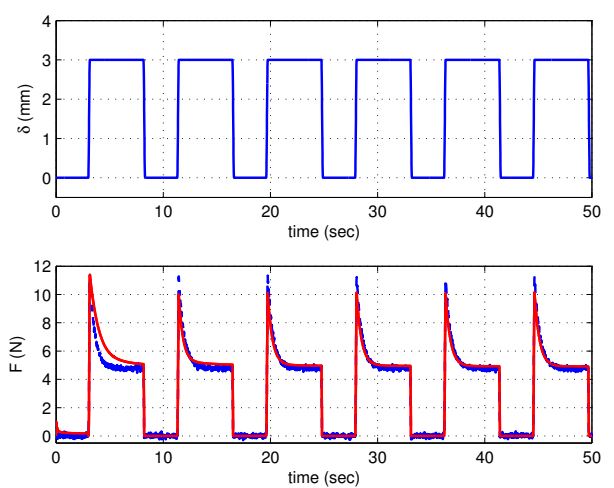

Fig. 18. Contact with a layer of polyurethane gel: measured data (dashed) and estimated model (solid).

slow dynamics (e.g. biological tissues). In fig. 18 the estimation process described in Sec. 4.3 is performed, by considering the contact with a layer of polyurethane gel. In this case, the deformation has been applied according to a step (or, to be precise, as a feasible approximation of the step with a rising ramp performed at $30 \mathrm{~mm} / \mathrm{s}$ ) and there are not speed limitations during the contact (differently from Hunt-Crossley model, whose validity is limited under a certain velocity). As shown in fig. 19, the estimator converges rather quickly to a stable parameters configuration ${ }^{5}$ (for both the elastic response and the reduced relaxation function), which guarantees an optimal approxi-

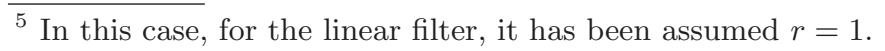



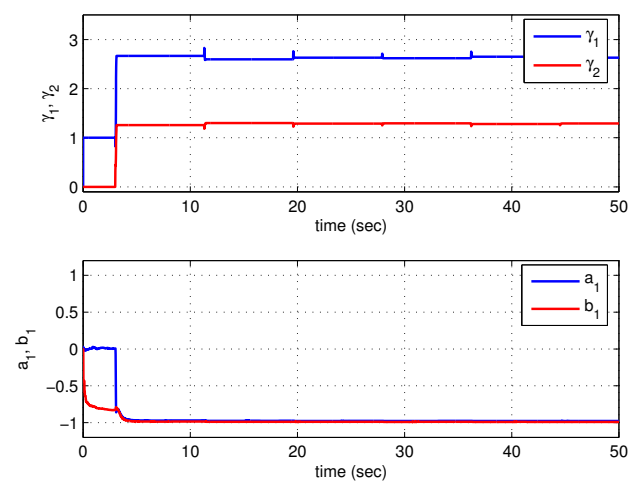

Fig. 19. Contact with a layer of polyurethane gel: parameters estimation.

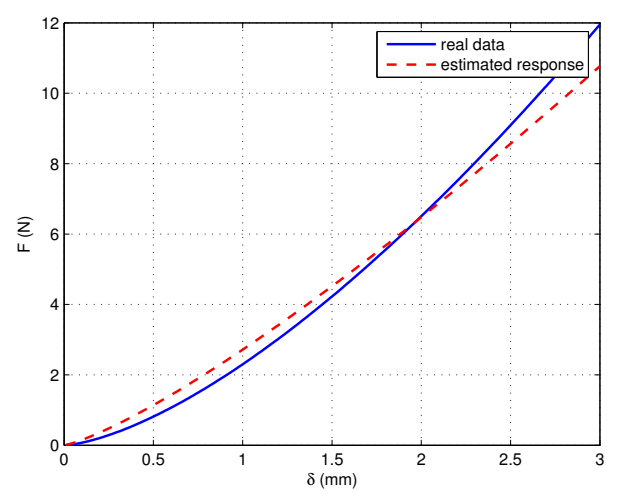

Fig. 20. Elastic response of polyurethane: real (dashed) and estimated (solid) response.

mation of the measurements. Finally in fig. 20, the comparison of the elastic response, as it has been estimated at the end of the identification process, with the real one is reported. Also in this case, the approximation can be considered satisfactory.

\section{Conclusions}

In this chapter, an overview of modelling and estimation techniques for nonlinear contact dynamics with hard and soft surfaces is given, with the purpose to use such results in telemanipulation schemes to improve their performances, and in particular the transparency towards the human operator. The 
proposed models are the Hunt-Crossley and the quasi-linear/Hammerstein model, both able to capture the nonlinearities characterizing the behavior (in particular the elasticity) of many materials during physical interactions. The former has been extensively used to describe the interaction of robot manipulators with the environment, the latter is very suitable to model the behaviors of visco-elastic materials (e.g. biological tissues), characterized by very slow dynamic modes.

For both models some suitable identification algorithms have been developed, which allows to estimate online linear and non-linear terms. Finally, the proposed estimation techniques are validated through some experimental tests. This kind of identification schemes can be profitably used for real-time impedance estimation in telemanipulation architectures. The extension to 3D models, for general applications with 6- degrees of freedom manipulators interacting with a remote environment, is therefore necessary and is a current research topic.

\section{References}

1. P. Arcara and C. Melchiorri. MISTRAL: Methodologies and Integration of Subsystems and Technologies for Anthropic Robots and Locomotion, chapter Comparison and Improvement of Control Schemes for Robotic Teleoperation Systems with Time Delay. Springer-Verlag, 2003.

2. J.E. Colgate. Robust impedance shaping telemanipulation. IEEE Transactions on Robotics and Automation, 9(4), 1993.

3. S. Hirche and M. Buss. Passive position controlled telepresence systems with time delay. In American Control Conference, volume 1, Denver, USA, June 2003.

4. D. Erickson, M. Weber, and I. Sharf. Contact stiffness and damping estimation for robotic systems. Int. Jour. of Robotic Research, vol. 22, 2003.

5. K.H. Hunt and F.R.E. Crossley. Coefficient of restitution interpreted as damping in vibroimpact. ASME Journal of Applied Mechanics, pages 440-445, June 1975.

6. K. L. Johnson. Contact Mechanics. Prentice Hall International Editions, 1995.

7. W.N.Findley, J.S. Lai, and K. Onaran. Creep and Relaxation of Nonlinear Viscoelastic Materials. North-Holland Publishing Company, 1976.

8. S. Misra abd A. M. Okamura. Environment parameter estimation during bilaterl telemanipulation. In Symposium on Virtual Interfaces for Virtual Environment and Teleoperator Systems, Alexandria, Virginia, USA, March 2006.

9. Y. Yokokohji and T. Yoshikawa. Bilateral control of master-slave manipulators for ideal kinesthetic coupling. In IEEE International Conference on Robotics and Automation, Nice, France, May 1992.

10. B. Hannaford. A design framework for teleoperators with kinesthetic feedback. IEEE Transactions on Robotics and Automation, 5(4), 1989.

11. G. De Gersem, H. Van Brussel, and J. Vander Sloten. Enhanced haptic sensitivity for soft tissues using teleoperation with shaped impedance reflection. In World Haptics Conference, Pisa, Italy, March 2005. 
12. K. Hashtrudi-Zaad and S.E. Salcudean. Adaptive transparent impedance reflecting teleoperation. In IEEE International Conference on Robotics and Automation, Minneapolis, Minnesota, April 1996.

13. K. B. Shimoga and A. A. Goldenberg. Soft robotic fingertips - part ii: A comparison of construction materials. The Int. Journal of Robotic Research, 15(4), 1996.

14. H. C. Güler, N. Berme, and S. R. Simon. A viscoelastic sphere model for the representation of plantar soft tissue during simulations. Jour. of Biomechanics, 31:847-853, 1998.

15. M. Shibata and S. Hirai. Stability and graspability analysis in grasping task taking fingertip dynamics into consideration. In Proc. of IEEE Int. Conf. on Intelligent Robots and Systems, IROS'04, Sendai, Japan, 2004.

16. K.H. Hunt and F.R.E. Crossley. Coefficient of restitution interpreted as damping in vibroimpact. ASME Journal of Appl. Mech., 1975.

17. D.W. Marhefka and D.E. Orin. A compliant contact model with nonlinear damping for simulation of robotic systems. IEEE Trans. on Systems, Man and Cybernetics, 29(6), 1999.

18. P.R. Kraus and V. Kumar. Compliant contact models for rigid body collisions. In Proc. of IEEE Int. Conf. on Robotics and Automation, ICRA' '7, 1997.

19. N. Diolaiti, C. Melchiorri, and S. Stramigioli. Contact impedance estimation for robotic systems. IEEE Trans. on Robotics and Automation, 21(5), 2005.

20. Y.C. Fung. Biomechanics: Mechanical Properties of Living Tissues. SpringerVerlag, 1993.

21. W. N. Findley and J. S. Y. Lay. A modified superposition principle applied to creep of non-linear viscoelastic material under abrupt changes in state of combined stress. Trans. of the Society of Rheology, vol. 11(3):361-380, 1967.

22. A. C. Pipkin and T. G. Rogers. A non-linear integral representation for viscoelastic behaviour. Jour. of Mech. Phys. Solids, vol. 16, 1968.

23. L. Ljung and T. Södertström. Theory and Practice of Recursive Identification. The MIT Press, Cambridge, Massachusetts, 1983.

24. K.J. Hunt, M. Munih, N. Donaldson, and F.M.D. Barr. Investigation of hammertein hypothesis in the modelling of electrically stimulated muscle. IEEE Transactions on Biomedical Enginering, 45(8), 1998.

25. E. Eskinat, SH. Johnson, and W.L. Luyben. Use of hammerstein models in identification of nonlinear systems. AIChE Journal, 37(2), 1991.

26. K.S. Narendra and P.G. Gallman. An iterative method for the identification of the nonlinear systems using the hammerstein model. IEEE Transactions on Automatic Control, 12, 1966.

27. I. Kao and F. Yang. Stiffness and contact mechanics of soft fingers in grasping and manipualtion. IEEE Trans. on Robotics and Automation, 20(1), 2004.

28. L. Biagiotti, C. Melchiorri, P. Tiezzi, and G. Vassura. Modelling and identification of soft pads for robotic hands. In Proc. of IEEE Int. Conf. on Intelligent Robots and Systems, IROS'05, 2005. 